\title{
Ratio of $J / \psi$ Production Cross Sections in Deep Inelastic Muon Scattering from Tin and Carbon
}

\author{
THE NEW MUON COLLABORATION (NMC)
}

\begin{abstract}
Bielefeld University ${ }^{1+}$, CERN ${ }^{2}$, Freiburg University ${ }^{3+}$, Max-Planck Institute für Kerphysik, Heidelberg ${ }^{4+}$, Heidelberg University ${ }^{5+}$, Mainz University ${ }^{6+}$, Mons University ${ }^{7}$, Neuchatel University ${ }^{8}$, NIKHEF- $K^{9++}$, University of California, Santa Cruz ${ }^{10}$, Paul Scherrer Institute ${ }^{11}$, Torino University and INFN Torino ${ }^{12}$, Uppsala University ${ }^{13}$, Institute for Nuclear Studies, Warsaw ${ }^{14 *}$, Warsaw University ${ }^{15 * *}$
\end{abstract}

P. Amaudruz ${ }^{11 a)}$, M. Arneodo ${ }^{12}$, A. Arvidson ${ }^{13}$, B. Badelek ${ }^{15}$, G. Baum ${ }^{1}$, J. Beaufays ${ }^{9 b)}$, I.G. Bird ${ }^{4 c)}$, M. Botje ${ }^{12}$, C. Broggini ${ }^{8 d)}$, W. Brückner ${ }^{4}$, A. Brüll ${ }^{3}$, W.J. Burger ${ }^{11 e)}$, J. Ciborowski ${ }^{15}$, R. van Dantzig ${ }^{9}$, H. Döbbeling ${ }^{4 f)}$, J. Domingo ${ }^{11 g)}$, J. Drinkard ${ }^{10}$, H. Engelien ${ }^{3}$, M.I. Ferrero ${ }^{12}$, L. Fluri ${ }^{8}$, D. von Harrach ${ }^{4 i)}$, M. van der Heijden ${ }^{9}$, C. Heusch ${ }^{10}$, Q. Ingram ${ }^{11}$, K. Janson ${ }^{13}$, M. de Jong ${ }^{9}$, E.M. Kabuß ${ }^{4 i)}$, R. Kaiser ${ }^{3}$, T.J. Ketel ${ }^{9}$, F. Klein ${ }^{6}$, S. Kullander ${ }^{13}$, U. Landgraf ${ }^{3}$, T. Lindqvist ${ }^{13}$, G.K. Mallot ${ }^{6}$, C. Mariotti ${ }^{12}$, G. van Middelkoop ${ }^{2,9}$, Y. Mizuno $\left.{ }^{4 j}\right)$, J. Nassalski ${ }^{14}$, D. Nowotny ${ }^{4 k}$, C. Peroni ${ }^{12}$, B. Povh $^{4,5}$, R. Rieger ${ }^{6}$, K. Rith ${ }^{4}$, K. Röhrich ${ }^{6 l)}$, E. Rondio ${ }^{15}$, L. Ropelewski ${ }^{15}$, A. Sandacz ${ }^{14}$, C. Scholz $^{4}$, U. Sennhauser ${ }^{11 m)}$, F. Sever ${ }^{1 n)}$, T.-A. Shibata ${ }^{5}$, M. Siebler ${ }^{1}$, A. Simon ${ }^{4}$, A. Staiano ${ }^{12}$, Y. Tzamouranis ${ }^{40)}$, J.L. Vuilleumier ${ }^{8}$, T. Walcher ${ }^{6}$, R. Windmolders ${ }^{7}$, F. Zetsche ${ }^{4}$

(Submitted to Nuclear Physics B)

\begin{abstract}
We present results on $J / \psi$ production in muon interactions with tin and carbon targets at incident muon energies of 200 and $280 \mathrm{GeV}$. The ratio of cross sections per nucleon for $J / \psi$ production on tin and carbon, $R(\mathrm{Sn} / \mathrm{C})$, is studied as a function of $p_{T}^{2}, z$ and $x$. We find an enhancement for coherent $J / \psi$ production $R_{c o h}(\mathrm{Sn} / \mathrm{C})=1.54 \pm 0.07$, a suppression for quasielastic production $R_{q e}(\mathrm{Sn} / \mathrm{C})=0.79 \pm 0.06$ and an enhancement for inelastic production $R_{\text {in }}(\mathrm{Sn} / \mathrm{C})=1.13 \pm 0.08$. The inelastic cross section ratio can be interpreted as an increase of the gluon distribution in tin with respect to that in carbon. The dependence of the ratio on $z$ and $p_{T}^{2}$ can explain the discrepancy between the results obtained in previous experiments.
\end{abstract}

\footnotetext{
${ }^{1}$ For footnotes see next page.
} 
+ Supported by Bundersministerium für Forschung und Technologie.

++ Supported in part by FOM, Vrije Universiteit Amsterdam and NWO.

* Supported by CPBP.01.06.

** Supported by CPBP.01.09.

a) Now at TRIUMF, Vancouver, BC V6T 2A3, Canada.

b) Now at Trasys, Brussels, Belgium.

c) Now at NIKHEF-K, P.O. Box 4395, 1009 AJ Amsterdam, The Netherlands.

d) Now at INFN, Laboratori Nazionali del Gran Sasso, 67010 Assergi, Italy.

e) Now at Université de Genève, 1211 Genève 4, Switzerland.

f) Now at GSI, 6100 Darmstadt, Germany.

g) Now at CEBAF, Newport News, VA 23606, U.S.A.

h) Now at UCLA, Los Angeles, CA 90024, U.S.A.

i) Now at University of Mainz, W-6500 Mainz, Germany.

j) Now at Osaka University, 567 Osaka, Japan.

k) Now at SAP AG, W-6909 Walldorf, Germany.

1) Now at IKP2-KFA, W-5170 Jülich, Germany,

m) Now at EMPA, 8600 Dübendorf, Switzerland.

n) On leave from Jozef Stefan Institut, Ljubljana, Yugoslavia, now at DPhN Saclay, 91191 Gif-sur-Yvette, France.

o) Now at University of Houston, TX 77204-5504, U.S.A. 


\section{Introduction}

The discovery of the EMC effect [1], that the structure function $F_{2}$ of a nucleon bound in a nucleus is different from that of a free nucleon, has stimulated a wide range of experimental [2,3] and theoretical activities [4]. It is important to determine whether such an effect also manifests itself in the gluon momentum distribution in the nucleon. This can be investigated by studying the production of the $J / \psi$ particle in deep inelastic scattering [5].

In the kinematic region where the $J / \psi$ is produced inelastically and incoherently (later referred to as inelastic) the virtual photon couples to the constituents of the nucleon. The Colour Singlet (CS) model [5] was found to give a good description of this production mechanism [6-9]. Within this model the inelastic cross section is proportional to the gluon momentum distribution so that the gluon distribution ratio for different nuclei can be obtained from the cross section ratio.

In the process of $J / \psi$ production in muon interactions with nuclei other contributions are present. The production cross section can be considered as the sum of the cross sections for coherent, quasielastic and inelastic interactions. In coherent $J / \psi$ production the recoiling nucleus emerges intact from the interaction, whereas in quasielastic production the interaction occurs elastically with a nucleon in the nucleus. We separated the kinematic regions where each of the above mentioned mechanisms is dominant to provide information on each of these processes. Since the mechanisms are different, one may expect the corresponding cross section ratios for heavy and light nuclei to be different. In the present paper we show that the seemingly inconsistent results obtained over the past fifteen years for such ratios can be reconciled.

\section{Experiment and Analysis}

The experiment was performed at the M2 muon beam line of the CERN SPS with a modified and upgraded version of the EMC forward spectrometer [10]. The data presented here were collected at two incident energies, 200 and $280 \mathrm{GeV}$.

A complementary target set-up was used, allowing measurements of cross section ratios of different target materials with small systematic errors. Each target set consisted of two tin and two carbon targets in alternate order. The complementary sets (see figure 1), where carbon and tin were interchanged, were alternately exposed to the beam. The tin targets were segmented such that the extent and the total amount of material along the beam were the same as for the carbon targets. Each target had a thickness of approximately $150 \mathrm{~g} / \mathrm{cm}^{2}$. Acceptances and integrated beam fluxes cancel in the calculation of the cross section ratio. The frequent exchange of the target sets also minimises the effects of any time dependence in the apparatus acceptance. A passive concrete absorber shielded the spectrometer from the electromagnetic and hadronic cascades originating from the interaction vertex. Data were collected using a specially designed trigger [8] which selected multi-muon tracks originating from the target. The information from the target calorimeters was not used.

The kinematics of the reaction was reconstructed from the measured momenta of both 
$J / \psi$ decay muons together with the incident and scattered muon. A special algorithm was devised to reconstruct tracks of particles scattered at very small angles and thus remaining in the beam region. Events with three or more outgoing muon tracks were selected for the analysis. For each event the hardware trigger requirements were checked using the reconstructed muon trajectories. To select the $J / \psi$ decay muons, the invariant mass of pairs of oppositely charged particles was calculated for the selected data sample. The track pair with an invariant mass closest to the $J / \psi$ rest mass was then taken. The scattered muon was selected from the other reconstructed tracks requiring the same charge as the incident muon. In $9 \%$ of the selected events more than one candidate for the scattered muon was found. These events were subjected to a further selection procedure in which the muon with the highest energy was chosen whenever its energy was at least $50 \%$ higher than the energy of any other candidate. Otherwise, the track with the smallest scattering angle was taken [7]. The kinematic variables used in the present analysis are listed in table 1. Cuts are applied in order to exclude regions of poor acceptance and high background contamination. These cuts are listed in table 2 for the two data samples.

Figure 1 shows the distribution of the reconstructed vertex positions along the beam direction. The vertex resolution was good enough to distinguish between the targets. The probability of associating events to the wrong material was found to be less than $1 \%$.

Figure 2 shows the distribution of the invariant mass $M_{\mu^{+} \mu^{-}}$of the selected muon pairs for tin and carbon for the $280 \mathrm{GeV}$ data sample. The sum of a gaussian distribution and an exponentially falling background was fitted to each of the mass spectra (smooth curves in the figure). Subtracting the fitted background from the number of events in the mass interval $2.7 \mathrm{GeV} / \mathrm{c}^{2} \leq M_{\mu^{+} \mu^{-}} \leq 3.5 \mathrm{GeV} / \mathrm{c}^{2}$, the final $J / \psi$ yields were found to be $514 \pm 30(\mathrm{Sn})$ and $416 \pm 24(\mathrm{C})$ events at $200 \mathrm{GeV}$ and $1353 \pm 46(\mathrm{Sn})$ and 1137 $\pm 40(\mathrm{C})$ events at $280 \mathrm{GeV}$. The fitted values of the peak positions are in agreement with the known value of the $J / \psi$ rest mass [11] (see table 3 ). The widths of the gaussian distributions are determined by the experimental momentum resolution and by multiple scattering in the targets and concrete absorber. This was verified by Monte Carlo studies.

A signal of $\psi^{\prime}$ production is visible in the mass spectrum of muon pairs from events originating in the passive absorber at $280 \mathrm{GeV}$ (see figure 3). For these events the mass resolution is better because of the reduced multiple scattering. Two gaussian distributions on top of an exponentially falling background were fitted simultaneously to the mass spectrum (smooth curve in the figure). Subtracting the fitted background from the number of events in the mass intervals $2.8 \mathrm{GeV} / \mathrm{c}^{2} \leq M_{\mu^{+} \mu^{-}} \leq 3.4 \mathrm{GeV} / \mathrm{c}^{2}$ and 3.4 $\mathrm{GeV} / \mathrm{c}^{2} \leq M_{\mu^{+} \mu^{-}} \leq 3.9 \mathrm{GeV} / \mathrm{c}^{2}$, the final $J / \psi$ and $\psi^{\prime}$ yields are $2415 \pm 58$ and $53 \pm$ 14 events, respectively. After dividing these yields by the corresponding branching ratios $\operatorname{BR}\left(J / \psi \rightarrow \mu^{+} \mu^{-}\right)=6.9 \pm 0.9 \%$ and $\operatorname{BR}\left(\psi^{\prime} \rightarrow \mu^{+} \mu^{-}\right)=0.77 \pm 0.17 \%$ [11], one obtains the cross section ratio $\sigma\left(\psi^{\prime}\right) / \sigma(J / \psi)=0.20 \pm 0.05$ (stat.) \pm 0.07 (syst.). The systematic error arises from the uncertainty in the branching ratios. The present result compares well with the value of ref. [12].

It was found earlier that the cross section per nucleon in deuterium is equal to that in hydrogen: $\sigma^{N}(D) / \sigma^{N}(H)=0.96 \pm 0.08$ [8]. The tin to carbon cross section ratio was therefore not corrected for the non-isoscalarity of the tin target, since such a correction would be less than $1 \%$. 


\section{Ratio of $J / \psi$ Production Cross Sections}

\subsection{Coherent $J / \psi$ Production}

In coherent $J / \psi$ production the recoiling nucleus emerges intact from the interaction. Since the nucleus carries away little energy most of the energy of the photon is transferred to the $J / \psi$, so that the energy fraction $z$ is close to unity.

Coherent photoproduction of vector mesons is usually interpreted in terms of Vector Meson Dominance (VMD) [13]. In the application of the VMD model to muoproduction of $J / \psi$ mesons it is assumed that the virtual photon couples to an off-shell $J / \psi$ meson which is put on-shell by diffractive scattering from a target nucleus. The total nuclear cross section for coherent production is usually taken to be of the form [13]

$$
\sigma_{c o h}\left(\gamma^{*} A\right)=\left(\frac{d \sigma\left(\gamma^{*} p\right)}{d t}\right)_{t=0} A^{2} \int_{t_{\min }}^{t_{\max }} F^{2}(-t) d t
$$

where $t=\left(q-p_{J / \psi}\right)^{2}$ with $p_{J / \psi}$ the four-momentum of the $J / \psi, t_{\min }=-\left(\frac{Q^{2}+M_{J / \psi}^{2}}{2 \nu}\right)^{2}$ at $z=1, t_{\max }$ is determined by the applied cuts, $A$ is the atomic number and $F(-t)$ is the form factor of the nucleus.

The calculation of $t$ involves the subtraction of two measured quantities of similar size. Consequently the resolution in $t$ is poor especially at small $t$ and the coherent peak arising from the form factor cannot be resolved. Since $p_{T}^{2}$ is measured much more accurately and $p_{T}^{2} \approx\left|t-t_{\min }\right|$ for $z$ close to unity, the $p_{T}^{2}$ distribution is used to study the coherent peak. Figure 4 shows the $p_{T}^{2}$ distributions for carbon and tin at 200 and $280 \mathrm{GeV}$ in the high $z$ region $(z \geq 0.9)$. The curves are fits to the four data sets with the sum of two exponentials:

$$
f\left(p_{T}^{2}\right)=a_{1} e^{-b_{1} p_{T}^{2}}+a_{2} e^{-b_{2} p_{T}^{2}} .
$$

The first term describes the low $p_{T}^{2}$ region where coherent production dominates and the second term the high $p_{T}^{2}$ region where the $J / \psi$ mesons are produced quasielastically. The results of the fits are given in table 4 . The peaks at small $p_{T}^{2}$, arising from coherent scattering from nuclei, are smeared by multiple scattering. The experimental $p_{T}^{2}$ resolution of $0.07(\mathrm{GeV} / \mathrm{c})^{2}$ does not allow a measurement of the intrinsic shapes of the $\mathrm{C}$ and $\mathrm{Sn}$ coherent peaks.

In order to obtain information on the coherent process, $p_{T}^{2} \leq 0.3(\mathrm{GeV} / \mathrm{c})^{2}$ was required for the high $z$ data $(z \geq 0.9)$. The quasielastic contribution in this region is given by the fit (second term in eq. 2) corrected by the suppression factor due to the Pauli exclusion principle [14]. This contribution affects the ratio by $3 \%$ and $10 \%$ for the 200 and 280 $\mathrm{GeV}$ data, respectively. The ratios obtained for the coherent cross sections per nucleon $R_{c o h}(\mathrm{Sn} / \mathrm{C})$ are given in table 5 , and are shown in figure 5 as a function of $\nu$. The curve represents the function:

$$
\frac{A_{S_{n}} \int_{t_{\max }}^{t_{\max }} F_{S n}^{2}(-t) d t}{A_{C} \int_{t_{\min }}^{t_{\max }} F_{C}^{2}(-t) d t},
$$


where the nuclear form factors $F_{S n}(-t)$ and $F_{C}(-t)$ are taken from [15]. Absorption effects of the $J / \psi$ are ignored. The ratio increases with $\nu$ since the value of $\left|t_{\text {min }}\right|$ decreases with $\nu$. A similar dependence can be seen in the data.

\subsection{Inelastic and Quasielastic $J / \psi$ Production}

Within the framework of the Colour Singlet model $[5,16,6-9]$ it is possible to extract the gluon momentum distribution $G(x)$ from the cross section for inelastic $J / \psi$ production. By measuring the cross section ratio of inelastic $J / \psi$ production from $\mathrm{Sn}$ and $\mathrm{C}$ it is then possible to obtain information on the ratio $G_{S_{n}}(x) / G_{C}(x)$ since the cross section is proportional to $G(x)$. The parameters of the model, the strong coupling constant $\alpha_{s}$, the $J / \psi$ leptonic width $\Gamma_{\mu^{+} \mu^{-}}$and the charmed quark mass $m_{c}$ cancel in this ratio. In order to apply the CS model in the region of its validity it is important to isolate a well defined sample of inelastic events according to $z$ and $p_{T}^{2}$.

Although the quasielastic events occur at $z$ near unity, the experimental resolution in $z$ is such that $z \leq 0.85$ is required to safely exclude the elastic contribution. This value was determined from Monte Carlo studies. The $p_{T}^{2}$ distributions for the inelastic events $(z \leq 0.85)$ are shown in figure 6 for $\mathrm{Sn}$ and $\mathrm{C}$ at 200 and $280 \mathrm{GeV}$. In the figure the $200 \mathrm{GeV}$ data were normalised to the $280 \mathrm{GeV}$ data such that the event yields from the concrete absorber were the same for the two energies. A comparison between figures 4 and 6 shows that the coherent contribution is present only at high $z$ and at low values of the transverse momentum. The inelastic $J / \psi$ signal is contaminated by $\psi^{\prime}$ production and its subsequent decay into $J / \psi$. The dashed line in figure 6 is the expected $p_{T}^{2}$ distribution of coherent $\psi^{\prime}$ production. At $p_{T}^{2}=0.4(\mathrm{GeV} / \mathrm{c})^{2}$ the contribution is of the order of $1 \%$, showing that the requirement $p_{T}^{2} \geq 0.4(\mathrm{GeV} / \mathrm{c})^{2}$ also removes the contamination from coherent $\psi^{\prime}$ decays. This contamination was estimated as follows. The coherent $\psi^{\prime}$ contribution is given by the first term of eq. 2 that fit the coherent $J / \psi$ signal multiplied by $\left[\sigma\left(\psi^{\prime}\right) / \sigma(J / \psi)\right] \cdot B R\left(\psi^{\prime} \rightarrow J / \psi\right)$; the cross section ratio is taken from section 2 and $B R$ from ref. [11].

The full line in figure 6 is the CS model prediction normalised to the data for $p_{T}^{2} \geq$ $0.4(\mathrm{GeV} / \mathrm{c})^{2}$. It can be seen from the figure that the model describes the inelastic data rather well. The contamination from inelastic $\psi^{\prime}$ production is proportional to the gluon momentum distribution according to the CS model. Its contribution to the inelastic $J / \psi$ signal then cancels in the ratio.

Figure 7 shows the ratio $R(\mathrm{Sn} / \mathrm{C})$ as a function of $z$ for high $p_{T}^{2}$ events $\left(p_{T}^{2} \geq 0.4\right.$ $\left.(\mathrm{GeV} / \mathrm{c})^{2}\right)$. For $z>0.85$ the cross section for $J / \psi$ production in $\mathrm{Sn}$ is smaller than that in $\mathrm{C}$, whereas for $z \leq 0.85$ it is larger. Increasing the $p_{T}^{2}$ cut does not affect the $z$ dependence of the ratio.

The quasielastic signals are given by the number of events in the region $0.9 \leq z \leq 1.1$ and $p_{T}^{2} \geq 0.4(\mathrm{GeV} / \mathrm{c})^{2}$; the inelastic signals in the region $z \leq 0.85$ and $p_{T}^{2} \geq 0.4(\mathrm{GeV} / \mathrm{c})^{2}$. The values for $R_{q e}(\mathrm{Sn} / \mathrm{C})$ and $R_{i n}(\mathrm{Sn} / \mathrm{C})$ are given in table 5 . The average value of $R_{i n}(\mathrm{Sn} / \mathrm{C})$ was found to be $1.13 \pm 0.08$. This can be interpreted as an enhancement of the gluon distribution in tin with respect to carbon. The ratio of the gluon distributions $R_{\text {in }}(\mathrm{Sn} / \mathrm{C})=G_{S n}(x) / G_{C}(x)$ as a function of $x$ is shown in figure 8 . The variable $x$ is the fraction of the nucleon momentum carried by the gluon and it is defined as [5, 16]: 


$$
x=\frac{1}{s}\left(\frac{M_{J / \psi}^{2}}{z}+\frac{p_{T}^{2}}{z(1-z)}\right) .
$$

Contamination from quasielastic $\psi^{\prime}$ production and subsequent decay into $J / \psi$ would increase the ratio $R_{\text {in }}(\mathrm{Sn} / \mathrm{C})$ under the assumption that the ratio for quasielastic $\psi^{\prime}$ production cross sections is the same as that for the $J / \psi$.

Different models [17-19] which describe shadowing in the nucleon structure function $F_{2}$ give predictions for the ratio of gluon distributions $G_{A}(x) / G_{D}(x)$. The common feature of these models is an enhancement by $3-8 \%$ of the gluon momentum distributions in the $x$ region covered by the present data $[0.02,0.2]$.

\section{Comparison with Previous Experiments}

The production of $J / \psi$ mesons has been previously studied with real and virtual photons both on hydrogen and on nuclear targets. At SLAC [20] $J / \psi$ production was measured on Be and Ta with real photons of $20 \mathrm{GeV}$ and the quasielastic cross section ratio was extracted. Another real photon experiment $\left(\mathrm{E}_{\gamma}=80-190 \mathrm{GeV}\right)$ was performed at FNAL on several nuclei [21]. The energy fraction $z$ was not measured and coherent effects were corrected for only by excluding events with $p_{T}^{2} \leq 0.15(\mathrm{GeV} / \mathrm{c})^{2}$. The only previous virtual photon experiments on different targets were performed at CERN by the EMC [22], which measured $J / \psi$ muoproduction on $\mathrm{H}$ and $\mathrm{D}$ (at $280 \mathrm{GeV}$ incident energy) and Fe (at 250 $\mathrm{GeV}$ incident energy). Cross sections were obtained over the full $z$ and $p_{T}^{2}$ ranges, with a correction for coherence in Fe in the region $z \geq 0.95$ and $p_{T}^{2} \leq 0.18(\mathrm{GeV} / \mathrm{c})^{2}$.

The present results, which were obtained in well defined kinematic regions, can now be compared with those obtained earlier although not always under identical conditions. The quasielastic ratio $R_{q e}(\mathrm{Ta} / \mathrm{Be})=0.83 \pm 0.05$ obtained at SLAC can be compared with the present value $(0.79 \pm 0.06)$ for $\mathrm{Sn} / \mathrm{C}$ at much higher photon energies. The production cross section ratio corrected for coherence effects as found at FNAL, R(Fe/Be $)=0.79 \pm 0.08$, can be reproduced from the present data by restricting $p_{T}^{2}$ to values larger than $0.4(\mathrm{GeV} / \mathrm{c})^{2}$ to exclude coherence and by integrating over the $z$ range accessible to our experiment [0.2,1.1]. By requiring the same energy domain, i.e. $80 \mathrm{GeV} \leq \nu \leq 190 \mathrm{GeV}$ and $E_{J / \psi} \geq$ $80 \mathrm{GeV}$, we find $R(\mathrm{Sn} / \mathrm{C})=0.82 \pm 0.06$ which is compatible with the FNAL value. A meaningful comparison with the EMC result [23] is not possible, since their resolution in $z$ and $p_{T}^{2}$ did not allow a complete subtraction of the coherent contribution. They found $R(\mathrm{Fe} /(\mathrm{H}+\mathrm{D}))=1.45 \pm 0.12$ (stat.) \pm 0.22 (syst.) which lies between our values for $R_{\text {in }}(\mathrm{Sn} / \mathrm{C})$ and $R_{\text {coh }}(\mathrm{Sn} / \mathrm{C})$.

\section{Summary and Conclusions}

We have measured the ratio of cross sections for $J / \psi$ production in deep inelastic muon scattering for tin and carbon. Our ability to separate the different kinematic regions permits the various results from previous experiments to be reconciled. The ratio $R(\mathrm{Sn} / \mathrm{C})$ of cross sections per nucleon is significantly larger than unity for coherent processes in 
which the nucleus emerges intact from the interaction. For quasielastic $J / \psi$ production the cross section per nucleon for tin is significantly lower than that for carbon. For inelastic $J / \psi$ production we found $R_{i n}(\mathrm{Sn} / \mathrm{C})=1.13 \pm 0.08$. This can be interpreted in the framework of the Colour Singlet model as an enhancement of the gluon distribution of tin with respect to carbon.

\section{ACKNOWLEDGEMENTS}

We wish to thank the technical staff of CERN and of the participating institutes for their invaluable contributions to the experiment. We are also grateful to N.N. Nikolaev and T. Sloan for fruitful discussions.

\section{References}

[1] EMC, J.J.Aubert et al., Phys.Lett. B123 (1983) 275.

[2] SLAC-E139, R.G.Arnold et al., Phys. Rev. Lett. 52 (1984) 727;

R.G.Arnold et al., SLAC-PUB-3257 (1983);

BCDMS, G. Bari et al., Phys. Lett. 163B (1985) 282;

BCDMS, A.C. Benvenuti et al., Phys. Lett. B 189 (1987) 483;

EMC-NA2', J. Ashman et al., Phys. Lett. B 202 (1988) 603;

EMC-NA28, M. Arneodo et al., Nucl. Phys. B333 (1990) 1;

NMC, P. Amaudruz et al., Z. Phys. C 51 (1991) 387.

[3] For a review of the experimental and theoretical situation see e.g. :

T. Sloan, G. Smadja and R. Voss, Phys. Rep. 162 (1988) 45.

[4] For a review of the theoretical models see e.g. :

L. Frankfurt and M. Strikman, Phys. Rep. 160 (1988) 235;

R.J.M. Covolan and E. Predazzi, in "Problems of Fundamental Modern Physics", Editors R. Cherubini, P. Dalpiaz and B. Minetti, World Scientific, Singapore (1991) p. 85.

[5] E.L.Berger and D.Jones, Phys. Rev. D23 (1981) 1521.

[6] EMC, N.Dyce, Ph.D thesis, Lancaster University (1988).

[7] NMC, D.Allasia et al., Phys. Lett. 258B (1991) 493.

[8] NMC, M.de Jong, Ph.D. thesis, Free University of Amsterdam (1991).

[9] NMC, C.Mariotti, proc. of the "Workshop on Hadronic Physics...", Dourdan 1990, to be published as a special issue of Nucl.Phys.A (1991). 
[10] NMC, D.Allasia et al., CERN/SPSC 85-18;

NMC, F. Zetsche, Ph.D. thesis, University of Heidelberg (1990), in German.

[11] Review of Particle Properties, Phys. Lett. B239 (1990).

[12] R.Barate et al., Z.Phys. C33 (1987) 505.

[13] T.H.Bauer et al., Rev.Mod.Phys. 50 (1978) 261;

E.Paul, Nucl.Phys. A446 (1985) 203.

[14] T.de Forest and J.D.Walecka, Adv. in Phys. 15 (1966) 1.

[15] I.Sick, Nucl.Phys. A218 (1974) 509;

I.Sick, Phys.Lett. 116B (1982) 212.

[16] A.D. Martin, C.-K.Ng and W.J.Stirling, Phys. Lett. B191 (1987) 200.

[17] F.E.Close, J.Qiu and R.G.Roberts, Phys.Rev. 40D (1989) 2820.

[18] U.Sukhatme, G.Wilk and K.E.Lassila, UICHEP-TH/90-10, (1990).

[19] L.L.Frankfurt, M.I.Strikman and S.Liuti, Phys. Rev. Lett. 65 (1990) 1725.

[20] R.L.Anderson et al., Phys.Rev.Lett. 38 (1977) 263.

[21] M.D.Sokoloff et al., Phys.Rev.Lett. 57 (1986) 3003.

[22] EMC, J.J.Aubert et al., Phys.Lett. 152B (1985) 433.

[23] EMC, J.J.Aubert et al., Nucl.Phys. B213 (1983) 1. 


$$
\begin{aligned}
& k=(E, \mathrm{k}) \\
& k^{\prime}=\left(E^{\prime}, \mathrm{k}^{\prime}\right) \\
& q=k-k^{\prime} \\
& q^{2}=-Q^{2} \\
& \nu=E-E^{\prime} \\
& s=M_{N}^{2}+2 M_{N} \nu-Q^{2} \\
& z=E_{J / \psi} / \nu \\
& p_{T}^{2}
\end{aligned}
$$

$\theta_{\text {decay }}$ 4-momentum of the incident muon

4-momentum of the scattered muon

4-momentum of the virtual photon

invariant mass squared of the virtual photon

energy of the virtual photon in the laboratory frame

the square of the photon-nucleon centre-of-mass energy

energy fraction carried by the $J / \psi$ in the laboratory frame

transverse momentum squared of the $J / \psi$ with respect to the photon direction

the angle of the positive decay muon in the helicity frame

Table 1: Kinematic variables

\begin{tabular}{|l|c|c|c}
\hline Variable & Lower Limit & Upper Limit & Units \\
\hline$E_{\mu}^{\prime}$ & 15 & & $\mathrm{GeV}$ \\
$E_{\text {decay } \mu}$ & 10 & & $\mathrm{GeV}$ \\
$\nu(E=200 \mathrm{GeV})$ & 40 & 180 & $\mathrm{GeV}$ \\
$\nu(E=280 \mathrm{GeV})$ & 60 & 240 & $\mathrm{GeV}$ \\
$Q^{2}$ & & 20 & $\mathrm{GeV}^{2}$ \\
$p_{T}^{2}$ & 0.2 & 10 & $(\mathrm{GeV} / \mathrm{c})^{2}$ \\
$z$ & -0.9 & 1.1 & \\
$\cos \left(\theta_{\text {decay }}\right)$ & 2.7 & 0.9 & \\
$M_{\mu^{+} \mu^{-}}$ & & 3.5 & $\mathrm{GeV} / \mathrm{c}^{2}$
\end{tabular}

Table 2: Cuts applied in the event selection

\begin{tabular}{c|c|c|c|}
\hline Target & $E(\mathrm{GeV})$ & $M_{0}\left(\mathrm{MeV} / \mathrm{c}^{2}\right)$ & $\sigma\left(\mathrm{MeV} / \mathrm{c}^{2}\right)$ \\
\hline $\mathrm{C}$ & 200 & $3090 \pm 8$ & $144 \pm 8$ \\
$\mathrm{Sn}$ & 200 & $3095 \pm 8$ & $136 \pm 9$ \\
\hline $\mathrm{C}$ & 280 & $3104 \pm 5$ & $137 \pm 5$ \\
$\mathrm{Sn}$ & 280 & $3092 \pm 6$ & $156 \pm 5$
\end{tabular}

Table 3: Results of the fits of the function $a e^{-\left(M_{\mu^{+} \mu^{-}}-M_{0}\right)^{2} / 2 \sigma^{2}}+b e^{-c M_{\mu^{+}} \mu^{-}}$to the mass spectra 


\begin{tabular}{l|c|c|c|c|c} 
Target & $E(\mathrm{GeV})$ & $a_{1}$ & $b_{1}(\mathrm{GeV} / \mathrm{c})^{-2}$ & $a_{2}$ & $b_{2}(\mathrm{GeV} / \mathrm{c})^{-2}$ \\
\hline $\mathrm{C}$ & 200 & $7.4 \pm 1.3$ & $9.4 \pm 1.9$ & $0.88 \pm 0.36$ & $1.40 \pm 0.24$ \\
Sn & 200 & $14.2 \pm 2.3$ & $11.2 \pm 1.8$ & $0.50 \pm 0.15$ & $0.83 \pm 0.14$ \\
& & & & & \\
$\mathrm{C}$ & 280 & $23.9 \pm 2.8$ & $8.9 \pm 1.2$ & $2.47 \pm 0.41$ & $1.15 \pm 0.09$ \\
Sn & 280 & $45.9 \pm 4.2$ & $11.5 \pm 1.0$ & $1.86 \pm 0.44$ & $1.18 \pm 0.17$
\end{tabular}

Table 4: Results of the fits of the function $a_{1} e^{-b_{1} p_{T}^{2}}+a_{2} e^{-b_{2} p_{T}^{2}}$ to the $p_{T}^{2}$ distributions of elastic $J / \psi$ production

\begin{tabular}{|c|c|c|c|}
\hline & $200 \mathrm{GeV}$ & $280 \mathrm{GeV}$ & combined \\
\hline $\begin{array}{c}\text { Coherent events } \\
z \geq 0.9, p_{T}^{2} \leq 0.3(\mathrm{GeV} / \mathrm{c})^{2}\end{array}$ & $1.43 \pm 0.14$ & $1.58 \pm 0.08$ & $1.54 \pm 0.07$ \\
\hline $\begin{array}{c}\text { Quasielastic events } \\
z \geq 0.9, p_{T}^{2} \geq 0.4(\mathrm{GeV} / \mathrm{c})^{2}\end{array}$ & $0.94 \pm 0.14$ & $0.73 \pm 0.07$ & $0.79 \pm 0.06$ \\
\hline $\begin{array}{c}\text { Inelastic events } \\
z \leq 0.85, p_{T}^{2} \geq 0.4(\mathrm{GeV} / \mathrm{c})^{2}\end{array}$ & $1.10 \pm 0.18$ & $1.14 \pm 0.10$ & $1.13 \pm 0.08$ \\
\hline
\end{tabular}

Table 5: Ratio $R(\mathrm{Sn} / \mathrm{C})$ of the cross sections per nucleon for $J / \psi$ production in the different kinematic regions 


\section{FIGURE CAPTIONS}

Figure 1: The target set-up and the distribution of the reconstructed vertex positions along the beam direction summed over both target positions.

Figure 2: Invariant mass distributions of selected $\mu^{+} \mu^{-}$pairs from tin and carbon at $280 \mathrm{GeV}$ incident muon energy. The smooth curves are fits to the observed distributions (see text).

Figure 3: Invariant mass distribution of selected $\mu^{+} \mu^{-}$pairs from the concrete absorber at $280 \mathrm{GeV}$ incident muon energy. The smooth curve is discussed in the text.

Figure 4: The $p_{T}^{2}$ distributions of the high $z(z \geq 0.9) J / \psi$ signal for carbon and tin at 200 and $280 \mathrm{GeV}$ incident muon energies. The solid (dashed) curves are fits of eq.2 to the observed distributions of $\mathrm{C}(\mathrm{Sn})$.

Figure 5: Ratio of coherent $J / \psi$ production cross sections per nucleon $R_{\text {coh }}(\mathrm{Sn} / \mathrm{C})$ as a function of $\nu$, for 200 and $280 \mathrm{GeV}$ incident muon energies. The curve is discussed in the text.

Figure 6: The $p_{T}^{2}$ distributions of the low $z(z \leq 0.85) J / \psi$ signal for carbon and tin at 200 and $280 \mathrm{GeV}$ incident muon energies. The smooth curves are the CS model predictions normalised to the data. The dashed curves represent the expected coherent $\psi^{\prime}$ contributions.

Figure 7: The measured cross section ratio per nucleon $R(\mathrm{Sn} / \mathrm{C})$ as a function of the energy fraction $z$ for events with $p_{T}^{2} \geq 0.4(\mathrm{GeV} / \mathrm{c})^{2}$.

Figure 8: $G_{S n}(x) / G_{C}(x)$ as a function of the fraction $x$ of the nucleon momentum carried by the gluon for $z \leq 0.85$ and $p_{T}^{2} \geq 0.4(\mathrm{GeV} / \mathrm{c})^{2}$. 


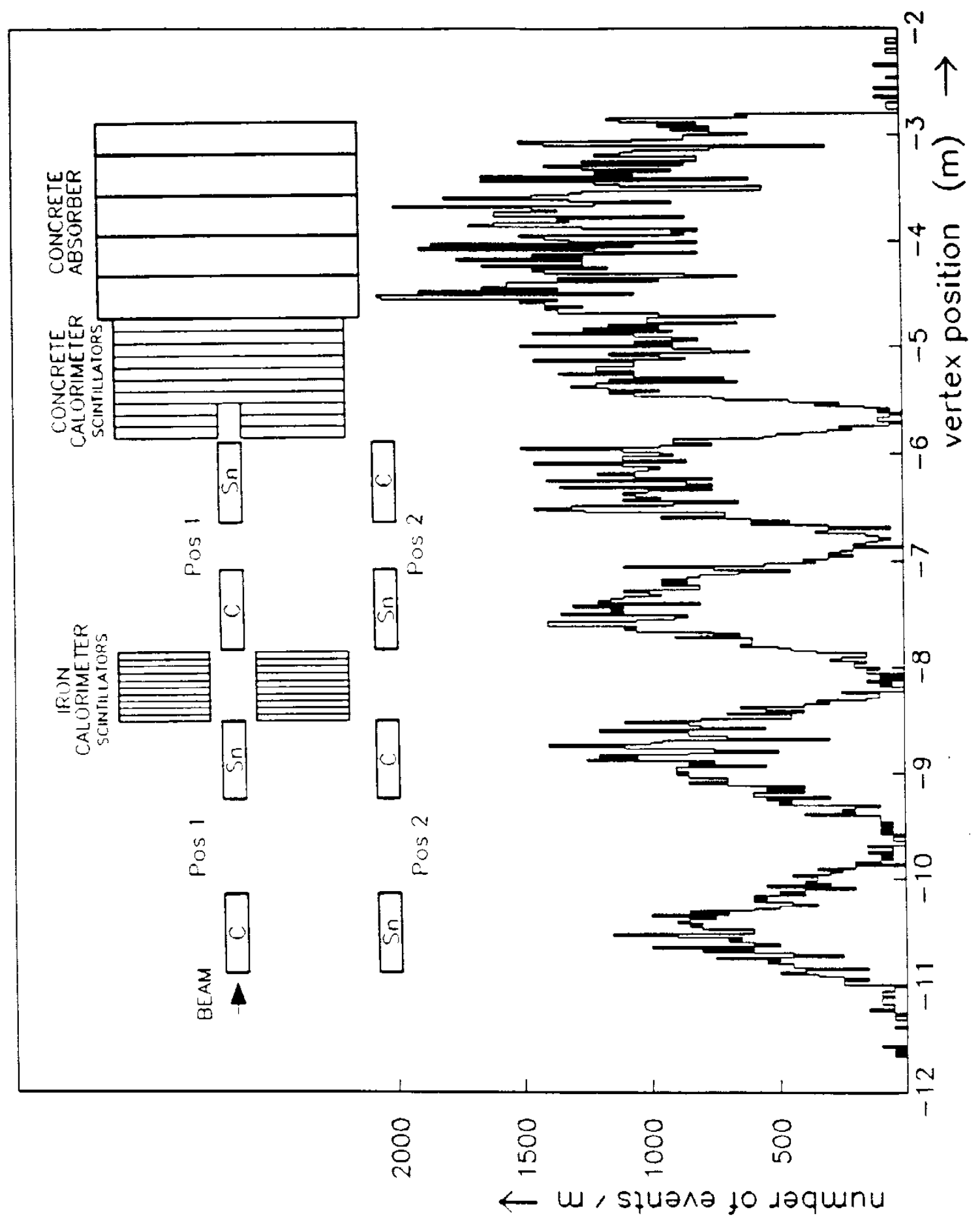

Fig. 1 


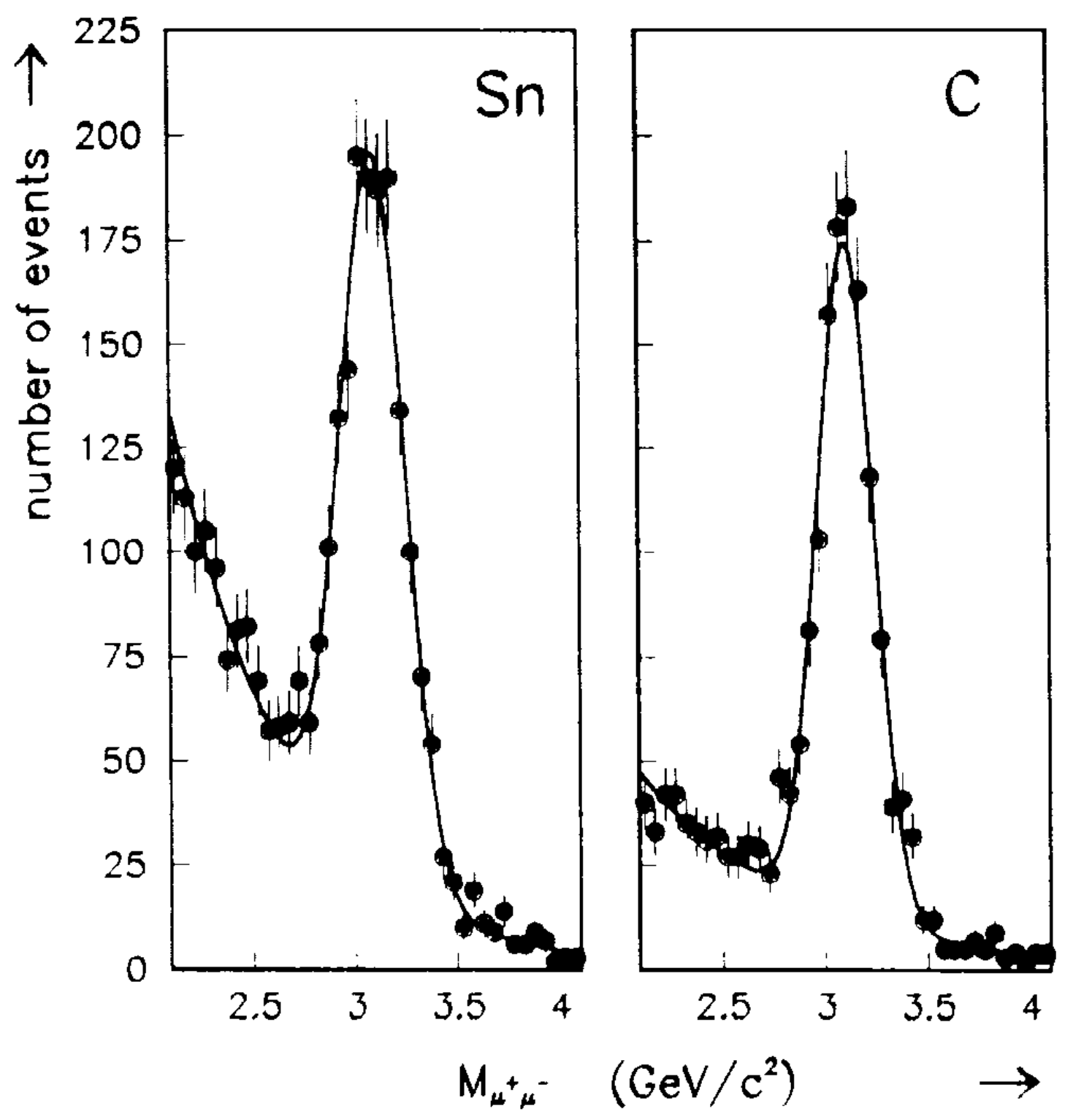

Fig. 2 


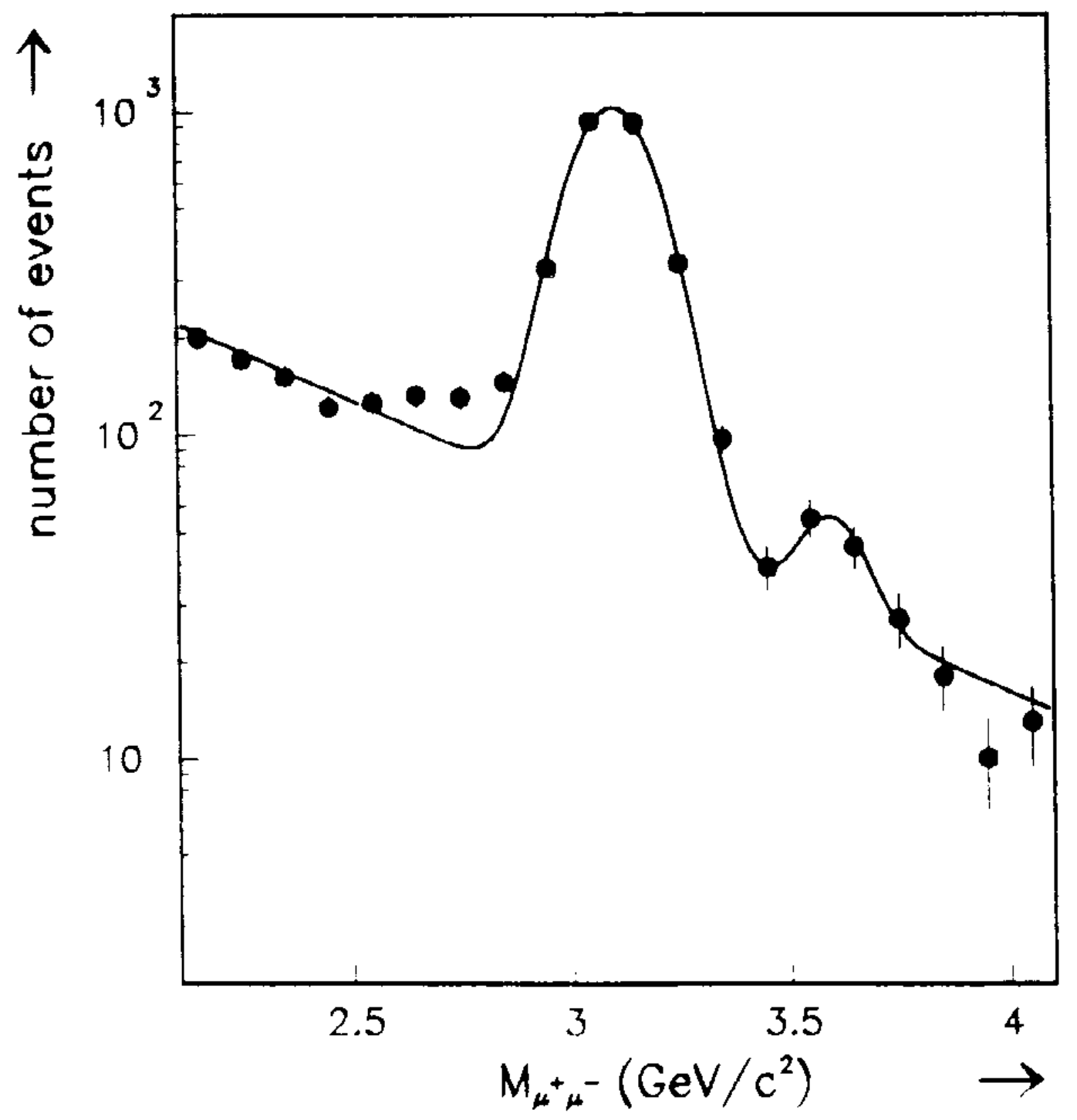

Fig. 3 

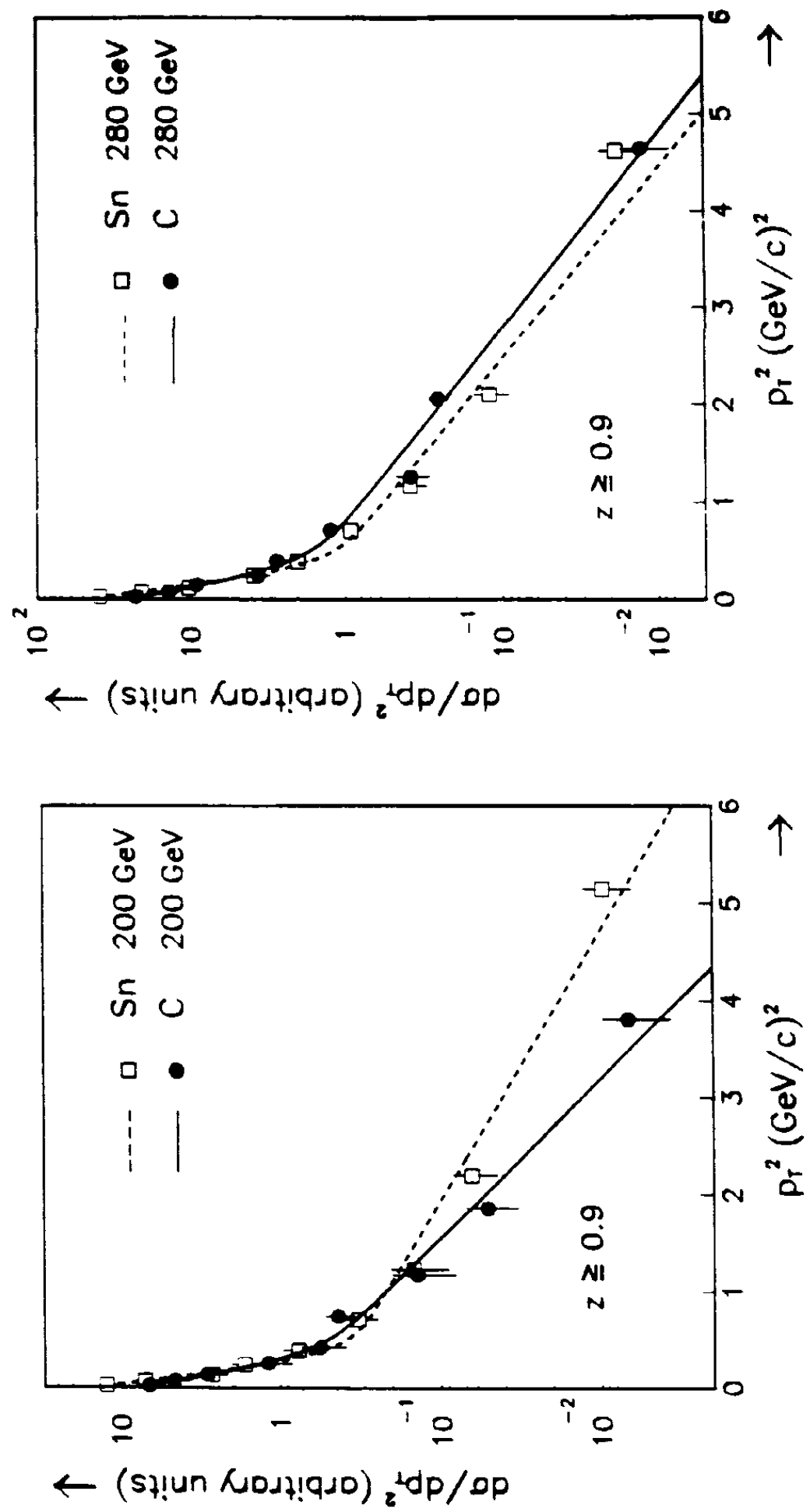

Fig. 4 


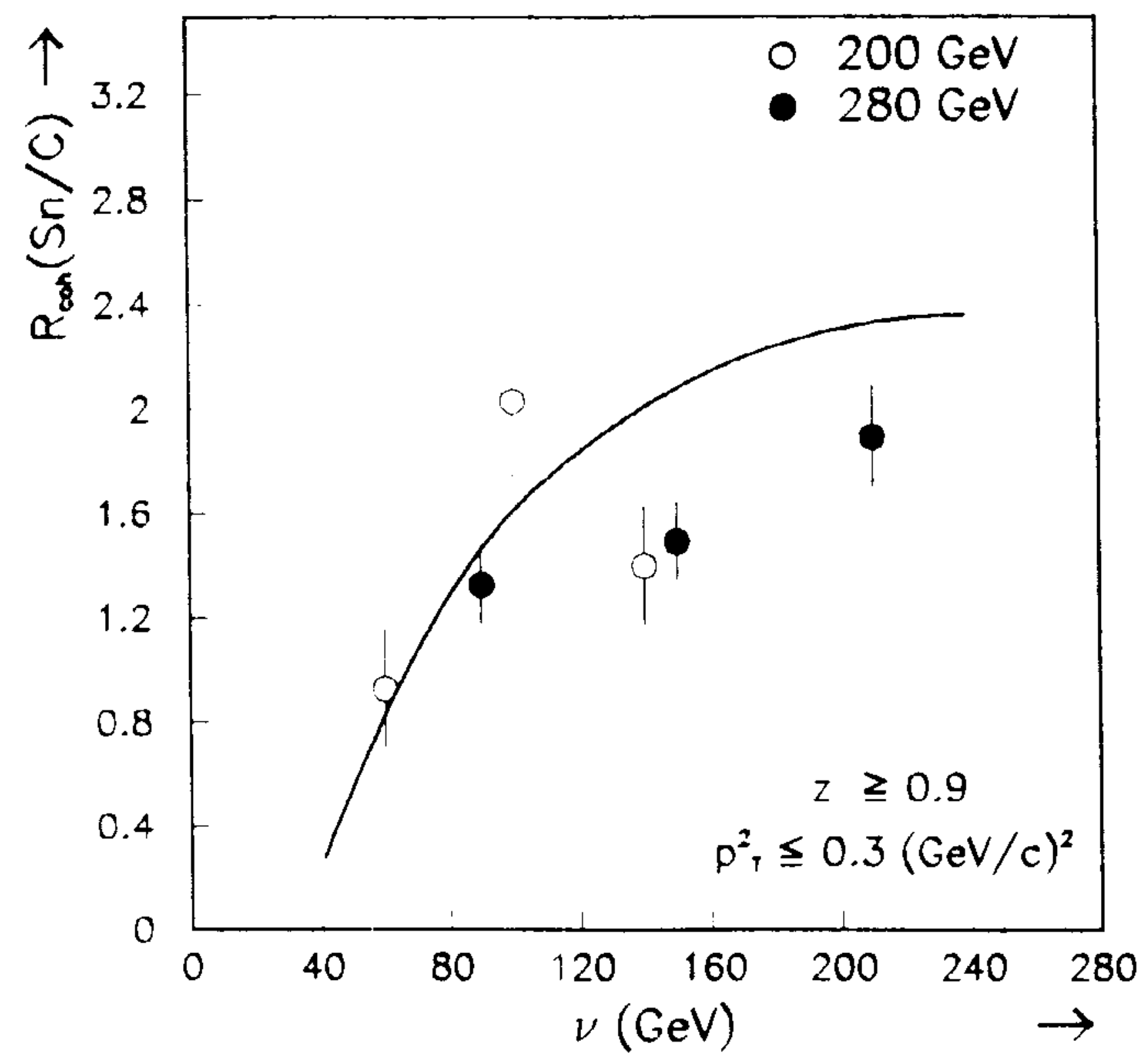

Fig. 5 

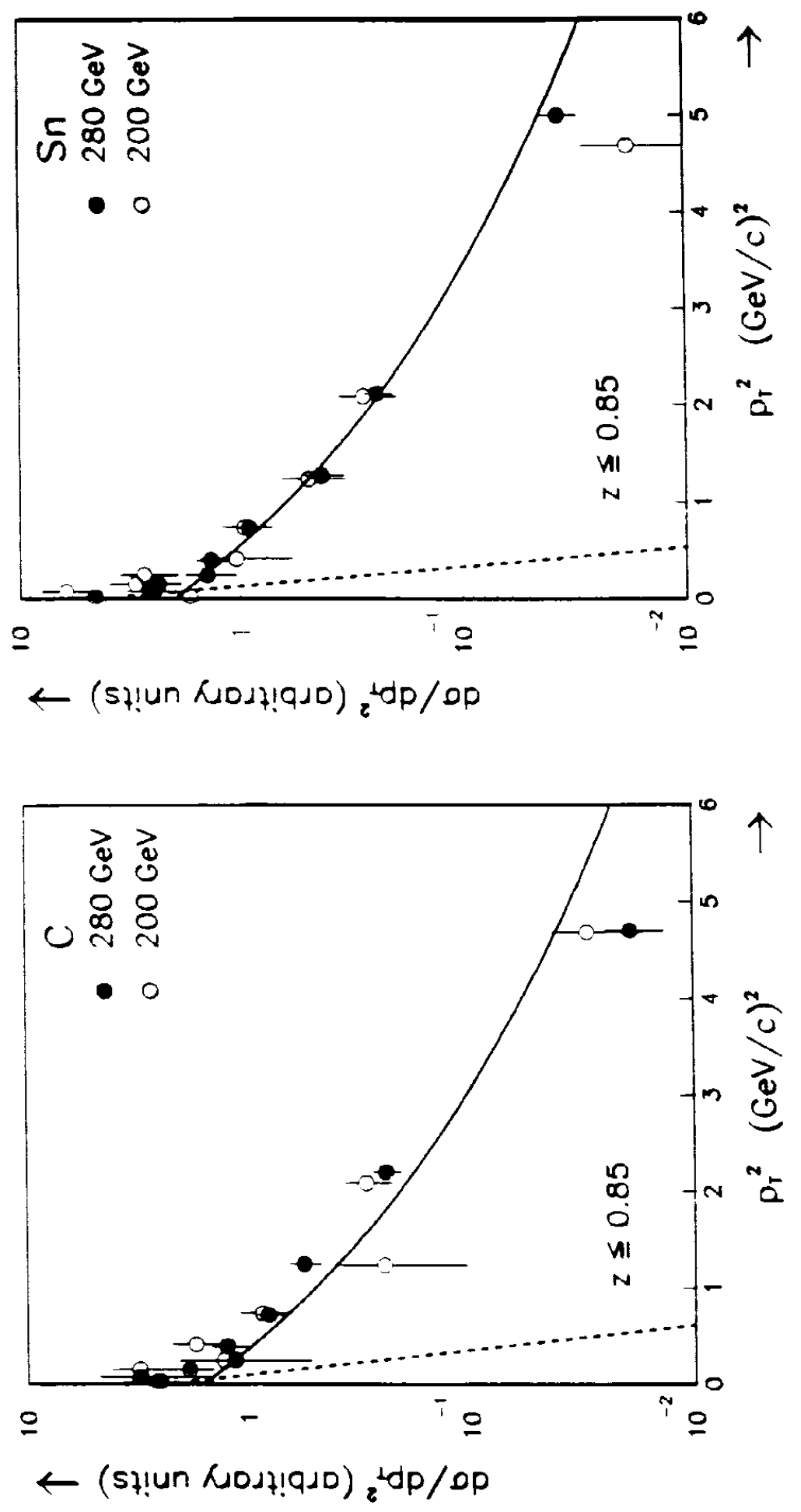

Fig. 6 


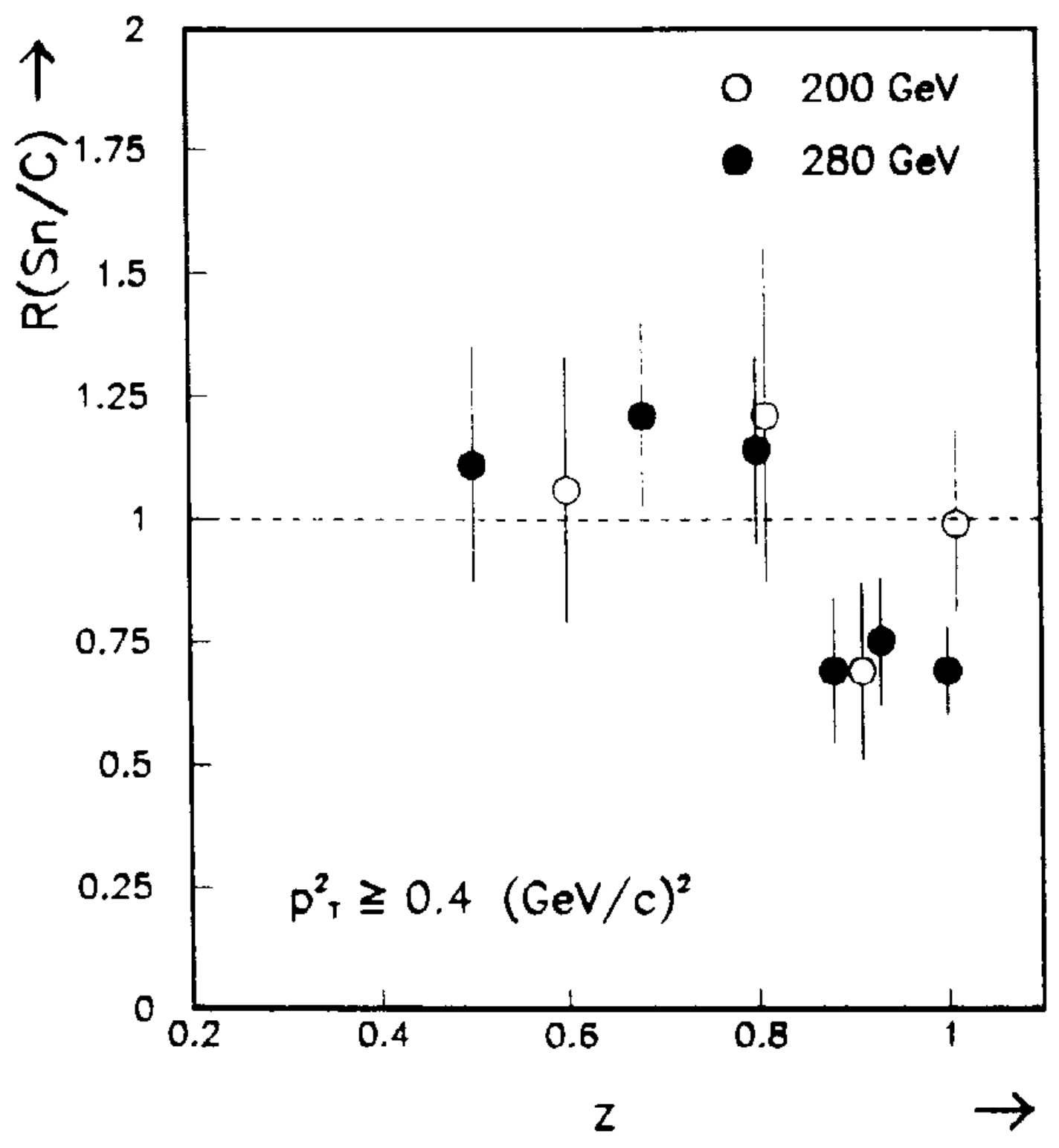

Fig. 7 


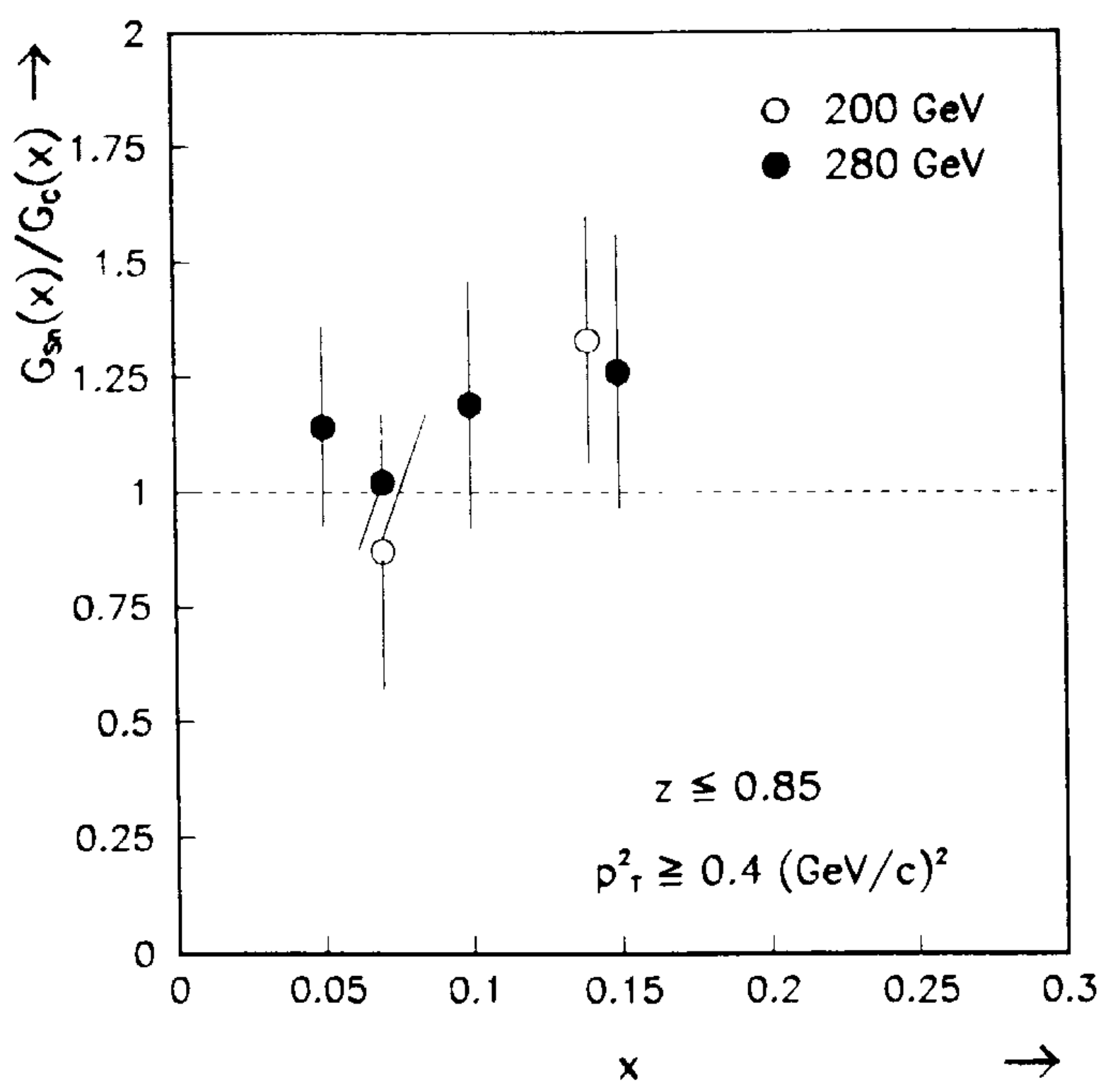

Fig. 8 